\title{
Analytical solution of neutron difussion equation for a PWR typical lattice
}

H. M. Nour and G. Abu-Zaied 


\title{
ANALYTICAL SOLUTION OF NEUTRON DIFFUSION EQUATION FOR A PWR TYPICAL LATTICE
}

\author{
H. M. NOUR AND G. ABU-ZAIED
}

[Received: September 12, 2003]

\begin{abstract}
This paper introduces an analytical technique to obtain the two-group neutron flux distribution on a typical square cell in the symmetrical core of a pressurised water reactor, PWR. Mathematically, the flux distribution is a solution to a system of fourth order homogeneous diffusion equations with irregular boundary conditions in Cartesian and polar coordinates. The formulae for the solution, obtained explicitly in terms of hypergeometric functions, are suitable for computer programming leading to temperature distribution and thermal stress analysis on fuel rod, etc. The proposed technique is capable of calculating the neutron flux at the corners of the typical cell, where neither approximated equivalent cell methods nor numerical methods succeeded. An actual condition of steady state critical reactors is studied, and numerical results by the proposed technique are compared to those of an approximated equivalent cylindrical cell technique.
\end{abstract}

Mathematics Subject Classification: 35k57

Keywords: Nuclear fuel cell, analytical solution, neutron diffusion equation, irregular boundary conditions

\section{InTRODUCTION}

Many engineering applications are simulated mathematically as partial differential equations with nonsmooth irregular boundary conditions, described geometrically by a composite of different shapes such as triangles, rectangles, and circles [1-3], as in the theory of elasticity [4-6] or in neutron diffusion [7,8]. Most of the customary numerical methods such as finite difference, finite elements and nodal methods fail at the corners as being singular points [9-12]. Also equivalent boundaries methods such as cylindrical $[13]$ or hexagonal $[14,15]$ are affected by corners ignorance. Approximate analytical methods obtain the solution as an infinite series [16], which is exposed to round off and truncation errors, and require stability and convergence investigation of the calculations.

One of the most powerful ways of treating multi-region reactors is by the group diffusion method. In this method the energies of all the neutrons are divided into 
a number of energy groups. The neutrons within each group are then lumped together and their diffusion, scattering, absorption, and other interactions are described in terms of suitably averaged diffusion coefficients and cross-sections, which are collectively known as group constants.

The simplest group diffusion problem involves only one group of neutrons, which, for simplicity, are assumed to be all thermal neutrons. A more accurate procedure, particularly for thermal reactors, is to split the neutrons into two groups; in which case, thermal neutrons are included in one group called the thermal or slow group, and all the other neutrons are included in the fast group. This approach was utilised in [13] assuming only one-dimensional composite problem. In this work, the approach used in [13] is extended to two dimensions. Moreover, the fuel rod and the moderator are treated as separate regions as will be indicated in the following section.

It must be emphasised that the two-group method is not an exact technique for handling reactor criticality calculations [17]. Nevertheless, it does provide a relatively simple way of obtaining reasonable estimates of the critical mass, flux distributions, and so on.

\section{Problem statement}

2.1. Governing equations. Consider a two-region thermal reactor consisting of homogeneous fuel rods submerged in moderator, which is divided into symmetrical cells. Every cell is a fuel lump consisting of a rod with circular cross-section of radius $a$ in a square moderator with side length $2 b$. Due to the symmetry of the system, the cell can be divided into triangular regions and confine the solution to one single triangle, say $O A B$, as shown in Figure 1.

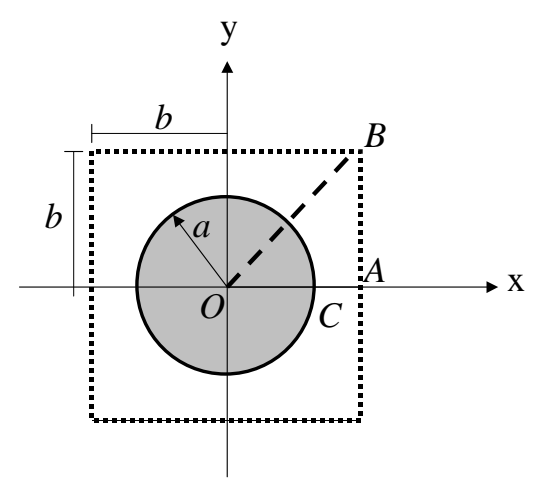

FIGURE 1. PWR typical lattice.

The steady state diffusion equation for the fast neutron flux in the fuel region $\phi_{1}^{F}$ is given by

$$
-D_{1}^{F} \nabla^{2} \phi_{1}^{F}+\left(\Sigma_{a 1}^{F}+\Sigma_{12}^{F}\right) \phi_{1}^{F}-v \Sigma_{f 1}^{F} \phi_{1}^{F}-v \Sigma_{f 2}^{F} \phi_{2}^{F}=0
$$


and that for the thermal flux $\phi_{2}^{F}$ in the fuel is described by

$$
-D_{2}^{F} \nabla^{2} \phi_{2}^{F}+\Sigma_{a 2}^{F} \phi_{2}^{F}-\Sigma_{12}^{F} \phi_{1}^{F}=0 .
$$

The diffusion equation for the fast neutron in the moderator region $\phi_{1}^{M}$ is

$$
-D_{1}^{M} \nabla^{2} \phi_{1}^{M}+\left(\Sigma_{a 1}^{M}+\Sigma_{12}^{M}\right) \phi_{1}^{M}=0,
$$

and for the thermal flux in the moderator $\phi_{2}^{M}$ is

$$
-D_{2}^{M} \nabla^{2} \phi_{2}^{M}+\Sigma_{a 2}^{M} \phi_{2}^{M}-\Sigma_{12}^{M} \phi_{1}^{M}=0,
$$

where

- $\nabla^{2}$ is the Laplace operator,

- $F$ and $M$ stand for fuel and moderator, respectively,

- subscripts 1 and 2 stand for fast and thermal fluxes, respectively,

- $D_{1}^{F}, D_{2}^{F}, D_{1}^{M}, D_{2}^{M}$ are diffusion coefficients for fuel and moderator in group 1 and 2 , respectively,

- $\Sigma_{a 1}^{F}, \Sigma_{a 2}^{F}, \Sigma_{a 1}^{M}, \Sigma_{a 2}^{M}$ are macroscopic absorption cross-sections,

- $\Sigma_{12}^{F}, \Sigma_{12}^{M}$ are macroscopic slowing down cross-sections,

- $\Sigma_{f 1}^{F}, \Sigma_{f 2}^{F}$ are macroscopic fission cross-sections,

- $v$ is the number of neutrons produced per fission.

The macroscopic cross-sections are assumed to be constants which characterise the material properties of the fuel and the moderator.

Since all cells are identical in an infinite uniform lattice, there can be no net flow of neutrons from one cell to another. It follows that the current density is zero along the boundary of each cell, also both fast and thermal fluxes and currents must be continuous at the fuel-moderator interface.

2.2. Boundary conditions. As mentioned in the previous section, both fast and thermal fluxes and currents must be continuous at the fuel-moderator interface, therefore the following conditions must be satisfied at $r=a$ :

$$
\begin{aligned}
\phi_{1}^{F} & =\phi_{1}^{M}, \\
\phi_{2}^{F} & =\phi_{2}^{M}, \\
D_{1}^{F} \frac{\partial \phi_{1}^{F}}{\partial r} & =D_{1}^{M} \frac{\partial \phi_{1}^{M}}{\partial r},
\end{aligned}
$$

and

$$
D_{2}^{F} \frac{\partial \phi_{2}^{F}}{\partial r}=D_{2}^{M} \frac{\partial \phi_{2}^{M}}{\partial r}
$$

Likewise, the symmetrical conditions for the triangular region $O A B$ in Figure 1 must be satisfied as follows: 
(i) Along the line $y=0$;

$$
\frac{\partial \phi_{j}^{i}}{\partial y}=0, \quad i=F, M, \text { and } \quad j=1,2
$$

(ii) Along the line $x=b$;

$$
\frac{\partial \phi_{j}^{M}}{\partial x}=0, \quad j=1,2
$$

(iii) Along the line $y=x$, where $0<x<b$

$$
\frac{\partial \phi_{j}^{i}}{\partial n}=0,
$$

where $n$ is the outward normal to the line $y=x$.

The major difficulty of this problem is that criticality conditions yield some of mathematical relations expressed in Cartesian coordinates and others expressed in polar coordinates.

On the one hand, expressing the entire problem in polar coordinates introduces some inaccuracy at the outer boundary of the moderator (at $x=b$ ), on the other hand, a mathematical statement in the Cartesian coordinates introduces inaccuracy at the interface of both the fuel and the moderator.

In the following section, these difficulties will be discussed in some detail in the frame of the proposed solution approach.

\section{AN APPROACH TO SOLUTION}

To find the fast and thermal fluxes which satisfy the criticality conditions of the reactor, it is necessary to solve the two-group equations for fuel and moderator, in the confined triangular region.

Beginning with the fuel, the coupled second-order differential equations (2.1) and (2.2) are decoupled by solving equation (2.1) for $\phi_{2}^{F}$ to get

$$
\phi_{2}^{F}=\frac{-1}{v \Sigma_{f 2}^{F}}\left(D_{1}^{F} \nabla^{2} \phi_{1}^{F}+\Sigma_{1}^{F} \phi_{1}^{F}\right) .
$$

Substituting this result into equation (2.2), one obtains a fourth-order equation for $\phi_{1}^{F}$ alone; namely,

$$
\tau_{f} L_{f}^{2} \nabla^{2} \nabla^{2} \phi_{1}^{F}-\left(\tau_{f}-L_{f}^{2}\right) \nabla^{2} \phi_{1}^{F}+\left(k_{\infty}-1\right) \phi_{1}^{F}=0,
$$

where

$$
\begin{gathered}
\Sigma_{1}^{F}=-\left(\Sigma_{a 1}^{F}+\Sigma_{12}^{F}-v \Sigma_{f 1}^{F}\right), \\
\tau_{f}=\frac{D_{1}^{F}}{\Sigma_{1}^{F}}, \quad L_{f}^{2}=\frac{D_{2}^{F}}{\Sigma_{a 2}^{F}}, \quad \text { and } \quad k_{\infty}=\frac{\Sigma_{12}^{F} v \Sigma_{f 2}^{F}}{\Sigma_{1}^{F} \Sigma_{a 2}^{F}},
\end{gathered}
$$


Equation (3.2) is a fourth-order differential equation with constant coefficients and can be factored to get

$$
\left(\nabla^{2}-\mu^{2}\right)\left(\nabla^{2}-\lambda^{2}\right) \phi_{1}^{F}=0
$$

where

$$
\mu^{2}=\frac{\left(\tau_{f}-L_{f}^{2}\right) \pm \sqrt{\left(\tau_{f}-L_{f}^{2}\right)^{2}-4 \tau_{f} L_{f}^{2}\left(k_{\infty}-1\right)}}{2 \tau_{f} L_{f}^{2}}
$$

and

$$
-\lambda^{2}=\mu^{2}-\frac{\tau_{f}-L_{f}^{2}}{\tau_{f} L_{f}^{2}}
$$

Equation (3.3) can be split into the following two equations

$$
\begin{aligned}
& \left(\nabla^{2}-\mu^{2}\right) X=0 \\
& \left(\nabla^{2}-\lambda^{2}\right) Y=0 .
\end{aligned}
$$

The general solution for $\phi_{1}^{F}$ is then a linear combination of the independent functions $X$ and $Y$; namely

$$
\phi_{1}^{F}=A X+C Y,
$$

where $A$ and $C$ are unknown constants. If the Laplace operator $\nabla^{2}$ in Cartesian form is used, then

$$
\begin{aligned}
& X(x, y)=(\cosh \alpha x)(\cosh \alpha y) \\
& Y(x, y)=(\cosh \beta x)(\cosh \beta y),
\end{aligned}
$$

where

$$
\alpha=\frac{\mu}{\sqrt{2}}, \text { and } \beta=\frac{\lambda}{\sqrt{2}}
$$

Having the fast flux in the fuel been determined, the thermal flux $\phi_{2}^{F}$ can be found from equation (3.1), in the form

$$
\phi_{2}^{F}=A S_{1} X+C S_{2} Y
$$

where

$$
S_{1}=\frac{\Sigma_{12}^{F} / \Sigma_{a 2}^{F}}{\mu^{2} L_{f}^{2}-1} \quad \text { and } \quad S_{2}=\frac{\Sigma_{12}^{F} / \Sigma_{a 2}^{F}}{\lambda^{2} L_{f}^{2}-1} .
$$

We now focus on the equations for fast neutron and thermal flux in the moderator. Equation (2.3) may be written in the form

$$
\nabla^{2} \phi_{1}^{M}-k_{1 M}^{2} \phi_{1}^{M}=0,
$$

where

$$
k_{1 M}^{2}=\frac{\Sigma_{a 1}^{M}+\Sigma_{12}^{M}}{D_{1}^{M}} .
$$


In general, the solution to equation (3.10) will be indicated by

$$
\phi_{1}^{M}=F Z_{1}(x, y),
$$

where $F$ is an unknown constant, and

$$
Z_{1}(x, y)=(\cosh \gamma(b-x))(\cosh \gamma(b-y)),
$$

where $\gamma=k_{1}^{M} / \sqrt{2}$. The thermal flux in the moderator $\phi_{2}^{M}$ can be found by assuming a solution to equation (2.4) in the form

$$
\phi_{2}^{M}=S_{3} \phi_{1}^{M}+G Z_{2},
$$

where $G$ is an unknown constant and

$$
S_{3}=\frac{\Sigma_{12}^{M} / D_{2}^{M}}{k_{2 M}^{2}-k_{1 M}^{2}}, \quad k_{2 M}^{2}=\frac{\Sigma_{a 2}^{M}}{D_{2}^{M}} .
$$

The function $Z_{2}(x, y)$ is then the solution of the equation

$$
\nabla^{2} Z_{2}-k_{2 M}^{2} Z_{2}=0
$$

whose solution may be written in the form

$$
Z_{2}(x, y)=(\cosh \omega(b-x))(\cosh \omega(b-y)),
$$

where $\omega=k_{2}^{M} / \sqrt{2}$.

The general solution which satisfies the symmetrical conditions (2.9) through (2.11), for the fast and thermal fluxes in both the fuel and moderator displayed in equations (3.6), (3.9), (3.11), and (3.13) can be conveniently written in the following matrix form:

$$
\left[\begin{array}{l}
\phi_{1}^{F} \\
\phi_{2}^{F} \\
\phi_{1}^{M} \\
\phi_{2}^{M}
\end{array}\right]=\left[\begin{array}{cccc}
1 & 1 & 0 & 0 \\
S_{1} & S_{2} & 0 & 0 \\
0 & 0 & 1 & 0 \\
0 & 0 & S_{3} & 1
\end{array}\right]\left[\begin{array}{c}
A X \\
C Y \\
F Z_{1} \\
G Z_{2}
\end{array}\right] .
$$

To calculate the four unknowns $A, C, F$, and $G$, criticality of the reactor in equations (2.5) through (2.8) is utilised.

In the preceding equations, the functions $X, Y, Z_{1}, Z_{2}$, are transformed into polar coordinates, then their magnitudes, and their partial derivatives are evaluated at the fuel-moderator interface where $r=a$.

Inserting the solutions of the fluxes, namely, equations (3.6), (3.9), (3.11), and (3.13), into equations (2.5) through (2.8) gives the following set of equations:

$$
\begin{gathered}
A X+C Y-F Z_{1}=0 \\
A D_{1}^{F} \frac{\partial X}{\partial r}+C D_{1}^{F} \frac{\partial Y}{\partial r}-F D_{1}^{M} \frac{\partial Z_{1}}{\partial r}=0, \\
A S_{1} X+C S_{2} Y-F S_{3} Z_{1}-G Z_{2}=0, \\
A D_{2}^{F} S_{1} \frac{\partial X}{\partial r}+C D_{2}^{F} S_{2} \frac{\partial Y}{\partial r}-F D_{2}^{M} S_{3} \frac{\partial Z_{1}}{\partial r}-G D_{2}^{M} \frac{\partial Z_{2}}{\partial r}=0 .
\end{gathered}
$$


In view of Cramer's rule regarding linear, homogeneous algebraic equations, the determinant of the coefficients multiplying the unknowns $A, C, F$, and $G$ is zero, then

$$
\left|\begin{array}{cccc}
X & Y & -Z_{1} & 0 \\
D_{1}^{F} \frac{\partial X}{\partial r} & D_{1}^{F} \frac{\partial Y}{\partial r} & -D_{1}^{M} \frac{\partial Z_{1}}{\partial r} & 0 \\
S_{1} X & S_{2} Y & -S_{3} Z_{1} & -Z_{2} \\
D_{2}^{F} S_{1} \frac{\partial X}{\partial r} & D_{2}^{F} S_{2} \frac{\partial Y}{\partial r} & -D_{2}^{M} S_{3} \frac{\partial Z_{1}}{\partial r} & -D_{2}^{M} \frac{\partial Z_{2}}{\partial r}
\end{array}\right|=0 .
$$

Equation (3.20) provides a relation, admittedly a somewhat complicated one, which must be satisfied if the reactor is to be critical. Criticality can be obtained by adjusting either the physical properties or the size of the reactor in such a way that the critical determinant given by (3.20) is precisely zero.

To find the fast and thermal fluxes it is necessary to determine the constants $A, C, F$, and $G$. It is not possible, however, to find the absolute values of all of these constants, since it can be shown that if the determinant of the coefficients in equation (3.20) is zero, as it must be for criticality, then these four equations are no longer independent. Therefore it is possible to determine only three of the constants in terms of $A$. Thus the absolute value of the flux cannot be found from these equations. While it is possible to specify the shape of the flux in a critical reactor, its magnitude depends upon the operating power of the system and is not determined by the group equations. To find the constant $A$ it is necessary to compute the reactor power $P$, where

$$
P=\kappa\left(\Sigma_{f 1}^{F} \int_{v} \phi_{1}^{F} d v+\Sigma_{f 2}^{F} \int_{v} \phi_{2}^{F} d v\right)
$$

It is found that

$$
\begin{aligned}
& C=\frac{A X}{\beta Y}\left(D_{1}^{F} \varkappa-D_{1}^{M} \xi\right), \\
& F=\frac{A X}{\beta Z_{1}}\left(D_{1}^{F} \varkappa-D_{1}^{M} \varepsilon\right),
\end{aligned}
$$

and

$$
G=\frac{A X}{\beta Z_{2}}\left(D_{1}^{F}\left(S_{2}-S_{3}\right) \varkappa+D_{1}^{F}\left(S_{2}-S_{3}\right) \varepsilon+D_{1}^{M}\left(S_{1}-S_{2}\right) \xi\right)
$$

where

$$
\beta=D_{1}^{M} \xi-D_{1}^{F} \varepsilon, \quad \varkappa=\frac{\frac{\partial X}{\partial r}}{X}, \quad \xi=\frac{\frac{\partial Z_{1}}{\partial r}}{Z_{1}}, \quad \text { and } \quad \varepsilon=\frac{\frac{\partial Y}{\partial r}}{Y} .
$$

All of the functions appearing in these equations are evaluated, at the fuel-moderator interface at $r=a$, (note that, numerically, it is preferable to consider $\theta=45^{\circ}$ ) where

$$
\begin{gathered}
\frac{\partial X}{\partial r}=\frac{\alpha}{2}\left[F_{1} \sinh \left(\alpha r F_{1}\right)+F_{2} \sinh \left(\alpha r F_{2}\right)\right] \\
\frac{\partial Y}{\partial r}=\frac{\beta}{2}\left[F_{1} \sinh \left(\beta r F_{1}\right)+F_{2} \sinh \left(\beta r F_{2}\right)\right] \\
\frac{\partial Z_{1}}{\partial r}=\frac{\gamma}{2}\left[-F_{1} \sinh \left(\gamma\left(2 b-r F_{1}\right)\right)+F_{2} \sinh \left(\gamma r F_{2}\right)\right]
\end{gathered}
$$


and,

$$
\frac{\partial Z_{2}}{\partial r}=\frac{\omega}{2}\left[-F_{1} \sinh \left(\omega\left(2 b-r F_{1}\right)\right)+F_{2} \sinh \left(\omega r F_{2}\right)\right]
$$

$$
F_{1}=\sin \theta+\cos \theta, \quad F_{2}=\sin \theta-\cos \theta .
$$

The solution written in the matrix form of equation (3.15) satisfies all conditions in (2.5) through (2.11) on the triangle region $O A B$, except only a part of condition (2.9) at the moderator region $(a \leq x \leq b)$. Along the sediment line $C A$, the normal slopes cannot be exactly determined but approximately

$$
\left[\frac{\partial \phi_{1}^{M}}{\partial y}\right]_{\substack{a \leq x \leq b, y=0}}=-\gamma F(\cosh \gamma(b-x))(\sinh \gamma b) \approx 0,
$$

also,

$$
\begin{aligned}
{\left[\frac{\partial \phi_{2}^{M}}{\partial y}\right]_{\substack{a \leq x \leq b, y=0}}=-\gamma S_{3} F(\cosh \gamma(b-x)) } & (\sinh \gamma b) \\
& -\omega G(\cosh \omega(b-x))(\sinh \omega b) \approx 0 .
\end{aligned}
$$

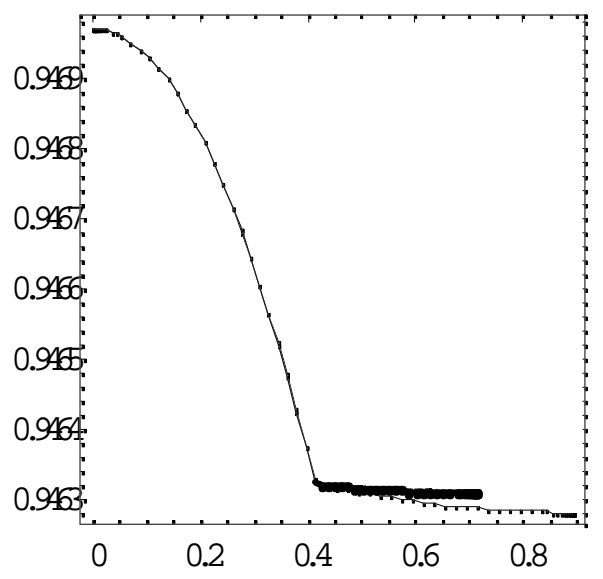

Figure 2. Fast flux distributions by the proposed and equivalent cylinder techniques.

\section{VAlidation}

The problem considered in this paper was treated in [16], where an approximate analytical solution was obtained. Another mathematical treatment based on an equivalent cylindrical cell was introduced in [13]. The solution obtained using the approach proposed in this work was validated by comparing some numerical results with solutions obtained by the above two methods. 


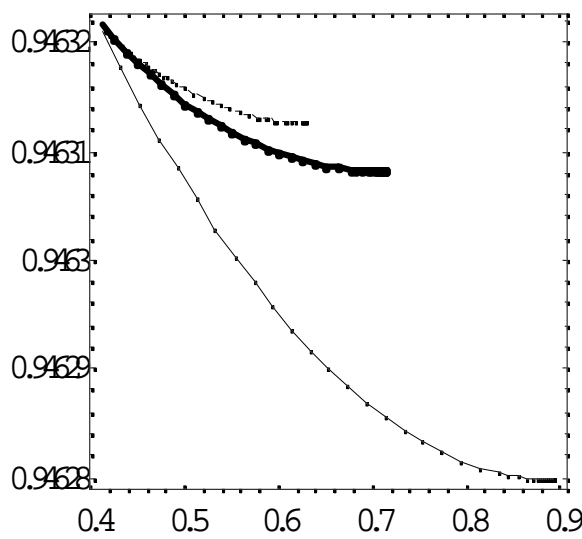

Figure 3. Fast flux distributions by the proposed and equivalent cylinder techniques, at moderator region.

Firstly, the approximated equivalent cylindrical cell technique, which is used now in reactors, is utilised to compare the numerical results of the obtained solution, as well as to compare the stability and convergence of the solution.

Secondly, the approximate analytical solution is introduced to show what the proposed solution is like in an explicit and simple form.

The approximate analytical and equivalent cylindrical cell solutions are highlighted in the following subsections.

4.1. Equivalent cylindrical cell. In the equivalent cylindrical cell approach, the actual cell which consists of a cylindrical fuel rod surrounded by a square moderator is replaced by a cylindrical cell of the same volume [13], see Figure 4.

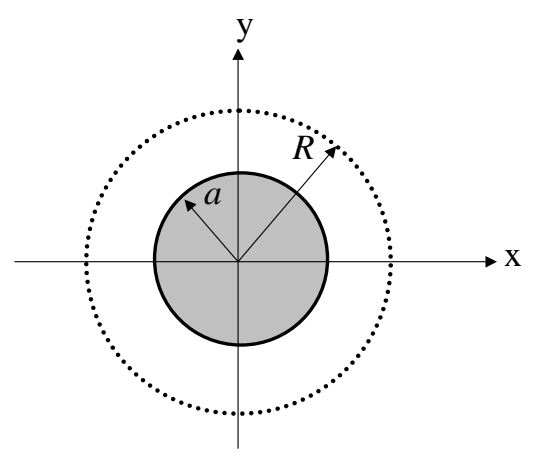

FIGURE 4. Equivalent cylindrical cell.

The solution of the system of equations (2.1) to (2.4) with the critical conditions (2.5) to (2.8), in piecewise cylindrical equivalent cell. The solution obtained may be 
recast into matrix form (3.15) as follows:

$$
\begin{gathered}
X=I_{0}(\mu r) \\
Y=I_{0}(\lambda r) \\
Z_{1}=K_{1}\left(k_{1 m} R\right) I_{0}\left(k_{1 m} r\right)+I_{1}\left(k_{1 m} R\right) K_{0}\left(k_{1 m} r\right) \\
Z_{2}=K_{1}\left(k_{2 m} R\right) I_{0}\left(k_{2 m} r\right)+I_{1}\left(k_{2 m} R\right) K_{0}\left(k_{2 m} r\right),
\end{gathered}
$$

where $R=\frac{2 b}{\sqrt{\pi}}$, and $I_{0}, I_{1}, K_{0}$, and $K_{1}$ are the Bessel functions.

4.2. Approximate analytical method. An approximate analytical method, which uses polar coordinates centred at the centre of the cell, was used in [16] for solving the system of equations given by (2.1) to (2.4). The solution is obtained as an infinite series of products of pairs of functions such that, for every pair, one of the functions depends solely on the angular variable while the other function depends on the radial variable and on the two energy groups. The solution is summarised in the matrix form (3.15) as

$$
\begin{gathered}
A X=\sum_{k=0}^{\infty} \cos 4 k \theta\left(a_{k} Z_{4 k}\left(B_{0} r\right)\right) \\
C Y=\sum_{k=0}^{\infty} \cos 4 k \theta\left(b_{k} Z_{4 k}\left(B_{1} r\right)\right) \\
F Z_{1}=\sum_{k=0}^{\infty} \cos 4 k \theta\left[c_{k} W_{4 k}\left(B_{2} r\right)+d_{k} Z_{4 k}\left(B_{2} r\right)\right] \\
G Z_{2}=\sum_{k=0}^{\infty} \cos 4 k \theta\left[e_{k} W_{4 k}\left(B_{3} r\right)+f_{k} Z_{4 k}\left(B_{3} r\right)\right],
\end{gathered}
$$

where $W_{4 k}$, and $Z_{4 k}$ are Bessel functions.

The coefficients $S_{1}, S_{2}, S_{3}, a_{k}, b_{k}, c_{k}, d_{k}, e_{k}$, and $f_{k}$ are determined from the boundary and critical conditions.

The results obtained from this solution are in an excellent agreement with our results. However, the solution form obtained using the proposed technique is expressed in a simpler and explicit form.

Each of the three techniques introduced above has its own arbitrary constants. To satisfy equivalence between two of them, arbitrary constants must be determined. One of the assumptions is that, at the cell centre, the equivalent techniques should have the same flux values.

Both the proposed technique and the equivalent cylindrical cell approach have the same flux values at the cell centre as:

$$
\left[\phi_{1}^{F}\right]_{r=0}=A+C,
$$


and

$$
\left[\phi_{2}^{F}\right]_{r=0}=A S_{1}+C S_{2}
$$

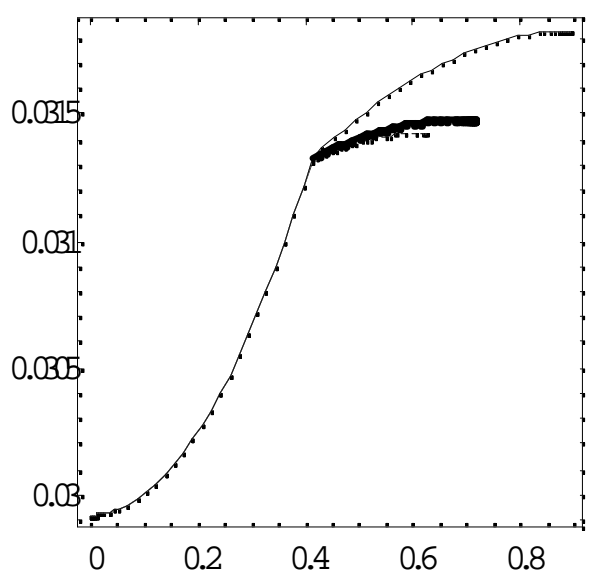

FIGURE 5. Thermal flux distributions by the proposed and equivalent cylinder techniques.

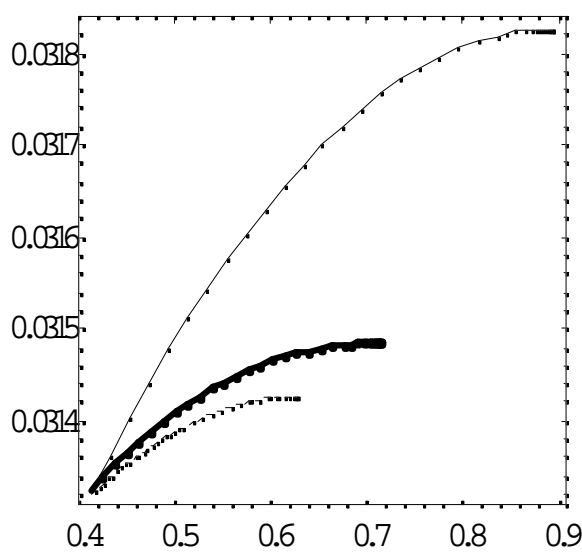

FIGURE 6. Thermal flux distributions by the proposed and equivalent cylinder techniques, at moderator region.

4.3. Study of a typical case. In a steady state symmetrical PWR typical lattice, twogroup constants are the following ones: 


$$
\begin{array}{llll}
D_{1}^{F}=1.13, & D_{2}^{F}=0.519, & D_{1}^{M}=21.2, & D_{2}^{M}=1.71, \\
\Sigma_{a 1}^{F}=0.0309, & \Sigma_{a 2}^{F}=0.283, & \Sigma_{a 1}^{M}=0.000127, & \Sigma_{a 2}^{M}=0.00432, \\
\Sigma_{12}^{F}=-0.00902, & \Sigma_{12}^{M}=0.0157, & \Sigma_{f 1}^{F}=0.0094, & \Sigma_{f 2}^{F}=0.211, \\
v=2.42, & a=0.4119, & b=0.6331 . &
\end{array}
$$

As a consequence, the equations of $\phi_{1}^{F}, \phi_{1}^{M}, \phi_{2}^{F}$, and $\phi_{2}^{F}$ for the proposed technique become (roughly):

$$
\begin{gathered}
\phi_{1}^{F}=\cosh (0.08 x) \cosh (0.08 y)-0.053 \cosh (0.52 x) \cosh (0.52 y), \\
\phi_{1}^{M}=0.95 \cosh (0.019(0.6331-x)) \cosh (0.019(0.6331-y)), \\
\phi_{2}^{F}=-0.033 \cosh (0.08 x) \cosh (0.08 y)+0.063 \cosh (0.52 x) \cosh (0.52 y),
\end{gathered}
$$

and

$$
\begin{aligned}
\phi_{2}^{M}=4.87 \cosh ( & 0.019(0.6331-x)) \cosh (0.019(0.6331-y)) \\
- & 4.84 \cosh (0.036(0.6331-x)) \cosh (0.036(0.6331-y)) .
\end{aligned}
$$

The normal derivatives in equations (3.25) and (3.26) are of order less than $10^{-10}$.

The corresponding solution sets for the equivalent cylindrical cell approach are

$$
\begin{gathered}
\phi_{1}^{F}=I_{0}(0.11 r)-0.053 I_{0}(0.73 r), \\
\phi_{1}^{M}=0.01\left(K_{1}(0.011) I_{0}(0.016 r)+I_{1}(0.011) K_{0}(0.016 r)\right), \\
\phi_{2}^{F}=-0.03 I_{0}(0.11 r)+0.06 I_{0}(0.73 r),
\end{gathered}
$$

and

$$
\begin{aligned}
\phi_{2}^{M}=0.056\left(K_{1}(0.011)\right. & \left.I_{0}(0.016 r)+I_{1}(0.011) K_{0}(0.016 r)\right) \\
& -0.1\left(K_{1}(0.021) I_{0}(0.029 r)+I_{1}(0.021) K_{0}(0.029 r)\right) .
\end{aligned}
$$

The numerical results are showed on the figures enclosed.

Figures 2 and 5 show that both fast and thermal fluxes $\left(\phi_{1}^{F}\right.$ and $\left.\phi_{2}^{F}\right)$ by the proposed or equivalent cylindrical cell techniques have the same distribution in fuel region.

Figures 3 and 6 show that, in the moderator region, both fast and thermal fluxes $\left(\phi_{1}^{M}\right.$ and $\left.\phi_{2}^{M}\right)$ by equivalent cylindrical cell technique take middle positions between $\theta=0^{\circ}$ and $\theta=45^{\circ}$.

Note 1. The dashed line stands for the flux at $\theta=0$, and the continuous one stands for the flux at $\theta=45^{\circ}$ by the proposed technique, while the thick line stands for the flux by the equivalent cylindrical cell technique.

Figure 7 specifies the fast flux $\left(\phi_{1}^{F}\right)$ distribution over the typical cell by the proposed technique, and Figure 8 specifies the fast flux distribution over the equivalent cylindrical cell.

Figures 9 and 10, respectively, describe the thermal flux distribution of the typical cell found by the proposed technique and the equivalent cylindrical cell. 


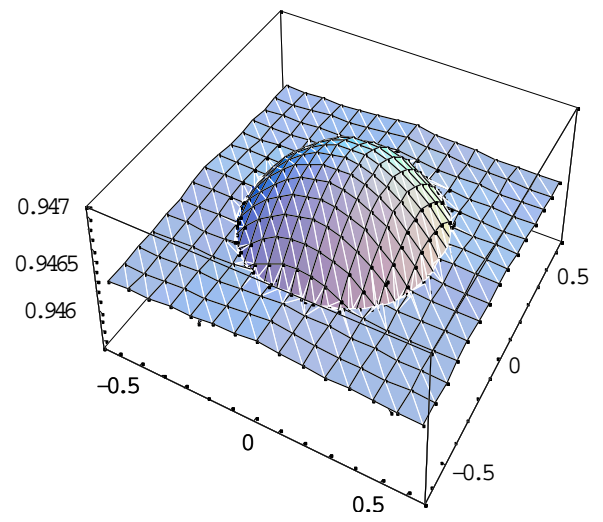

FIGURE 7. Fast flux distribution of the proposed technique.

Figures 11 and 12 show the variations of fast and thermal fluxes $\left(\phi_{1}^{M}, \phi_{2}^{M}\right)$, respectively, against the angle $\theta$, at different radii.

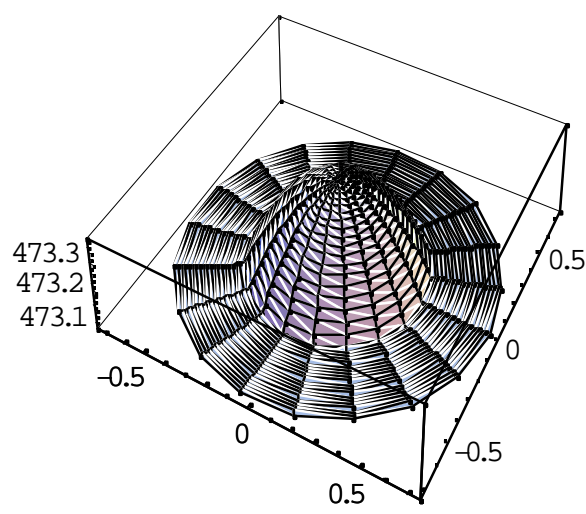

Figure 8. Fast flux distribution of equivalent cylinder technique.

\section{Conclusions}

An analytical technique was introduced for solving the two-group neutron diffusion equations in the critical reactor's typical lattice. Mathematically, it is a solution of the fourth order homogeneous diffusion equations with non-smooth (or irregular) boundary conditions in both the Cartesian and polar coordinates. The solution was obtained in the explicit form suitable for computer programming and other purposes such as analysis of temperature distribution, thermal stress on fuel rod, etc. By using this technique, one can compute the neutron flux at the corners of the typical cell, in 


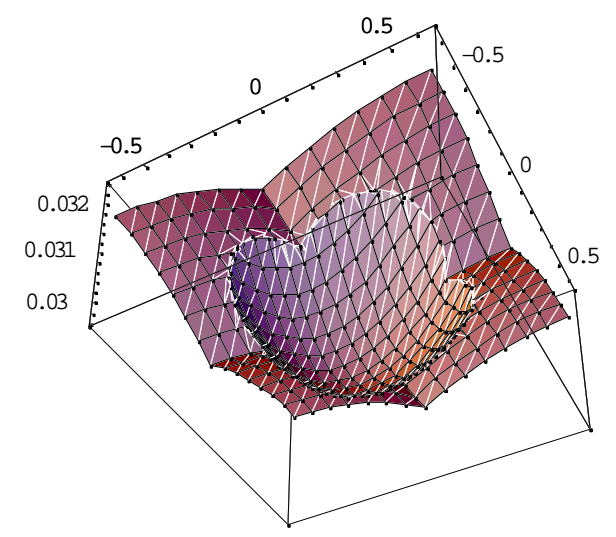

FiguRE 9. Thermal flux distribution of the proposed technique.

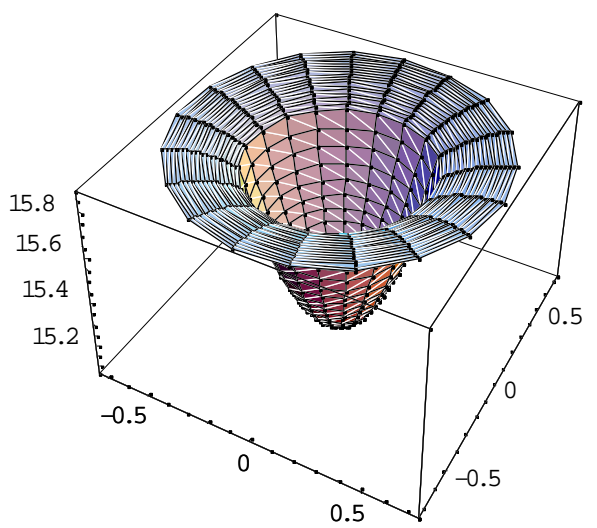

Figure 10. Thermal flux distribution of equivalent cylinder technique.

which case neither approximate equivalent cell methods nor numerical methods succeed. The proposed technique also avoids round off and truncation errors analysis and stability and convergence investigation in calculations, in which other approximate analytical methods may fail.

Future work:

(i) Studying temperature distribution of PWR typical lattice and stress analysis in fuel rod using the proposed technique.

(ii) Extending the proposed technique in three dimensions.

(iii) Studying flux distributions of non-symmetrical PWR typical lattice.

(iv) Treating the problem as a stochastic process, since the macroscopic crosssections are not constant but random variables. 


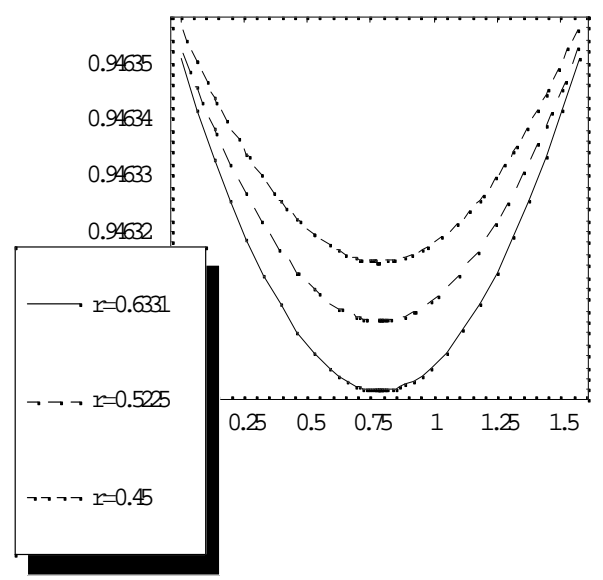

FIGURE 11. Variations of fast flux in moderator region against angle $\theta$, at different values of $r$.

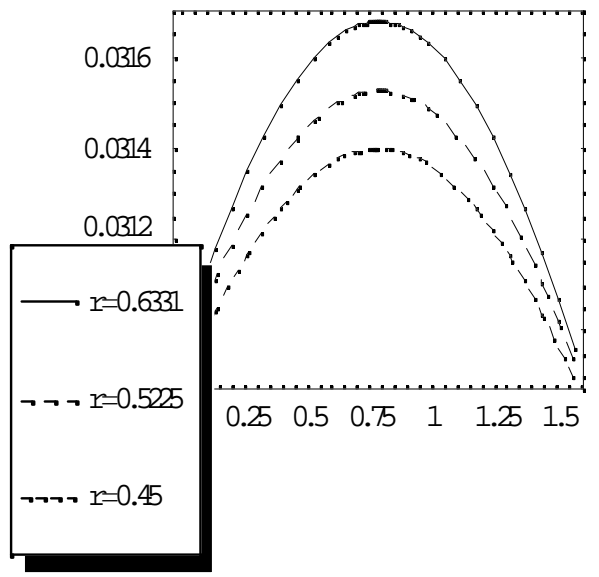

FiguRE 12. Variations of thermal flux in moderator region against angle $\theta$, at different values of $r$.

\section{REFERENCES}

[1] Grisvard, P., Elliptic problems in nonsmooth domains, Pitman, Boston, 1985.

[2] Grisvard, P., Wendland, W. L., ANd Whiteman, J. R.: Singularities and constructive methods for their treatment, Proc. Oberwolfach, Springer Verlag, New York, 1983.

[3] Babuska, I., Gus, B. Q., AND Osbord, J. E.: Regularity and numerical solution of eigenvalue problems with piecewise analytic data, SIAM J. Number. Anal., 26 (1989), No. 6, p. 1534.

[4] Sarkisyan, V. S. And Nour, H. M.: About a certain problem in the torsion theory of anisotropic rod, Ochinue Thabasic EGO, No. 2, Yerevan University, Armenia, 1993.

[5] Nour, H. M.: A suggested solution for torsion problem of homogeneous prismatic bar, Mansoura Engineering Journal (MEJ), 21 (1996), No. 4, 1-11. 
[6] Nour, H. M., Аtтia, G. M., And Авo El-MaAty, M. I.: Torsion on homogeneous anisotropic cylindrical prismatic bar, Alexandria Engineering Journal (AEJ), 37 (1998), No. 1D, 19-25.

[7] Cacuci, D. G.: Two-dimensional geometrical corner singularities in neutron diffusion. Part I: Analysis, Nuclear Science And Engineering, 128 (1998), 1-16.

[8] CAcucI, D. G.: Two-dimensional geometrical corner singularities in neutron diffusion. Part II: Application to the SNR-300 benchmark, Nuclear Science and Engineering: 128 (1998), 17-26.

[9] Hennart, J. P. and Mund, E. H.: Singularities in the finite element approximation of twodimensional diffusion problems, Nuclear Science And Engineering, 62 (1977), p. 55.

[10] Hennart, J. P., Malambu, E. M., Mund, E. H., and del Valle, E.: Efficient higher order nodal finite element formulations for neutron multigroup diffusion equations, Nuclear Science and Engineering, 124 (1996), 97-110.

[11] Joo, H. G. and Downar, T. J.: Stabilization techniques for the nonlinear analytic nodal method, Nuclear Science and Engineering, 130 (1998), 47-59.

[12] Sмгтн, K. S.: Nodal method storage reduction by non-linear iteration, Trans. Amer. Nucl. Soc., 44 (1983), p. 265.

[13] BARry, R., LEOPARD-A spectrum dependent non-spatial depletion code, WACP-3269-26 (1973).

[14] Chao, Y. A. AND Tsoulfanidis, N.: Conformal mapping and hexagonal nodal methods I: Mathematical foundation, Nuclear Science And Engineering, 121 (1995), 202-209.

[15] Chao, Y. A. and Shatilla, Y. A.: Conformal mapping and hexagonal nodal methods II: Implementation in the ANC-H code, Nuclear Science and Engineering, 121 (1995), 210-225.

[16] El-Awadi, I. AND Abu-Zaied, G.: Neutron diffusion in two-region domain with irregular boundaries, 12th International Mechanical Power Engineering Conference, IMPEC 12 (2001), pp. 1-13.

[17] Hennart, J. P., Malambu, E. M., Mund, E. H., and del Valle, E.: Efficient higher order nodal finite element formulations for neutron multigroup diffusion equations, Nuclear Science and Engineering, 124 (1996), 97-110.

\section{Authors' Addresses}

\section{H. M. Nour:}

Department of Mathematical and Physical Sciences, Mansoura University, Egypt

E-mail address: hanour@mans.edu.eg

\section{G. Abu-Zaied:}

Department of Mechanical Power Engineering, Faculty of Engineering, Mansoura University, EGYPT 